\title{
Nonstoichiometry and Weyl fermionic behavior in TaAs
}

\author{
J. Buckeridge, ${ }^{*}$ D. Jevdokimovs, C. R. A. Catlow, and A. A. Sokol \\ University College London, Kathleen Lonsdale Materials Chemistry, Department of Chemistry, 20 Gordon Street, \\ London WC1H OAJ, United Kingdom
}

(Received 2 September 2016; published 1 November 2016)

\begin{abstract}
The band structure of TaAs provides the necessary conditions for the emergence of Weyl fermions. Measurements verifying this fact are remarkably robust, given the reported levels of nonstoichiometry in typical single crystals. Here we demonstrate the surprising fact that a small degree of nonstoichiometry is essential for such observations in a wide range of temperatures. From first principles, we compute how crystal defects influence the position of the Fermi level relative to the so-called Weyl points, a key factor in allowing the detection of these particles. We show that observations of Weyl fermions depend crucially on nonstoichiometry and only occur within narrow ranges of elemental composition and temperature, indicating a considerable degree of fortuity in their discovery. Our approach suggests that in some cases the drive to produce ultra-pure crystals for measurements of exotic emergent phenomena may be misplaced.
\end{abstract}

DOI: 10.1103/PhysRevB.94.180101

Confirmation of the existence of a massless chiral spin$1 / 2$ quasiparticle, known as the Weyl fermion, an allowed solution to Dirac's equation [1-3], remained elusive until very recently $[4,5]$. Using angle-resolved photoemission spectroscopy (ARPES) and first-principles calculations, it has been shown [6] that due to spin-orbit effects on the energy bands, these particles arise in the Weyl semimetals TaP, TaAs, $\mathrm{NbP}$, and NbAs, of which TaAs is typically taken as the representative example [4,5,7-15]. The key feature in the electronic band structure is the Weyl node, a singularity point of Berry curvature close to the Fermi level, about which there is linear dispersion. These nodes appear as pairs of opposite chirality in bulk Weyl semimetals (dubbed $W 1$ and $W 2$ ) [7], resulting in "Fermi arcs" at the surface, which have been detected using ARPES. Moreover, associated properties such as negative magnetoresistance and ultra-high mobility have been observed $[16,17]$.

These discoveries were made using single crystals, typically grown by a chemical vapor transport method [18,19]. Crystal purity is rarely discussed, but there is strong evidence of a high defect concentration, with Ta deficiency present to some extent [20-22]. Moreover, different growth conditions, in terms of precursors, temperatures, and timings, will affect the balance of defects, in turn affecting the Fermi level, which must lie close to the Weyl nodes if Weyl fermion effects are to be detected. With this in mind, why these effects have been observed at all is somewhat puzzling. One explanation is the defects manifest as stacking faults, having a more benign effect on the Fermi level than point defects would [23]. Such stacking faults have been observed using scanning transmission electron microscopy on crushed crystals [23], but the mechanical stress used in the crushing procedure itself could promote formation

\footnotetext{
*j.buckeridge@ucl.ac.uk

Published by the American Physical Society under the terms of the Creative Commons Attribution 3.0 License. Further distribution of this work must maintain attribution to the author(s) and the published article's title, journal citation, and DOI.
}

of these faults, meaning that another explanation will be required for crystals at ambient conditions.

Off-stoichiometric defects in TaAs form on crystal growth, which is governed by the complicated binary phase diagram comprising stoichiometries that range from As-rich $\left(\mathrm{TaAs}_{2}\right)$ to As-poor $\left(\mathrm{Ta}_{5} \mathrm{As}_{4}, \mathrm{Ta}_{2} \mathrm{As}, \mathrm{Ta}_{3} \mathrm{As}\right)$ [20]. Producing TaAs crystals requires As-rich conditions at temperatures of $\sim 1000^{\circ} \mathrm{C}$ [24]. Different temperatures lead to different stoichiometries, but the phase relationship, key to explaining the observed nonstoichiometry, is not understood.

In this Rapid Communication we determine how nonstoichiometry affects the Fermi level relative to the $W 1$ and $W 2$ nodes in TaAs, and thus the observability of Weyl fermions. Using $a b$ initio calculations of the intrinsic point defect formation energies as a function of electron chemical potential, we compute the self-consistent Fermi level and hence the energy overlap of the charge carrier distribution with the Weyl nodes. We find that rather than always being detrimental, nonstoichiometry promotes Weyl fermion detection in specific temperature ranges, including those seen in experiment, indicating a level of fortuity in their discovery. It follows that nonstoichiometry is not only compatible with observing Weyl effects, but also necessary in a range of experimental conditions. Our approach can be adopted for other exotic phenomena that depend crucially on the Fermi level; we conclude that sometimes a drive for ultra-pure crystals may be misplaced.

Methods. To determine optimized structures and electronic properties, we have used density functional theory (DFT), as implemented in the VASP code [25-28] utilizing the solidscorrected Perdew-Burke-Ernzerhof (PBEsol) generalized gradient approximation exchange-correlation functional $[29,30]$, with the projector augmented wave method [31] to model the interaction between core and valence electrons (with five valence electrons for both $\mathrm{Ta}$ and As atoms). The TaAs primitive cell (space group $I 4_{1} m d$ ) was relaxed at a series of constant volumes, using a 400-eV plane-wave cutoff and a $9 \times 9 \times 9$ Monkhorst-Pack [32] $k$-point mesh, which provided convergence in the total energy up to $10^{-4} \mathrm{eV}$, fitting the resultant energy-volume data to the Murnaghan equation of state. Spin-orbit interactions were included where necessary [33] (more details can be found in Ref. [34]). To 
calculate the bulk density of states $\rho(E)$ as a function of energy $E$, we have performed single-point calculations on the relaxed primitive cell using an $18 \times 18 \times 18 k$-point mesh. We set $E=0$ at the VASP-computed Fermi level $\left(E_{F_{0}}\right)$ of the TaAs primitive cell.

To determine heats of formation, we have also relaxed primitive cells (space groups in parentheses) of $\mathrm{TaAs}_{2}(C 12 / m 1)$, $\mathrm{Ta}_{5} \mathrm{As}_{4}(I 4 / m), \mathrm{Ta}_{2} \mathrm{As}(P n n m)$, and $\mathrm{Ta}_{3} \mathrm{As}(B 2 / b)$ and computed their total energy [18-20]. In doing so, we have adopted the same approach and convergence criteria as that for TaAs.

We have considered the full range of intrinsic point defects in our study, i.e., $\mathrm{Ta}$ and $\mathrm{As}$ vacancies $\left(\mathrm{V}_{\mathrm{Ta}}\right.$ and $\mathrm{V}_{\mathrm{As}}$ respectively), interstitials $\left(\mathrm{Ta}_{i}\right.$ and $\mathrm{As}_{i}$ respectively), and antisites $\left(\mathrm{Ta}_{\mathrm{As}}\right.$ and $\mathrm{As}_{\mathrm{Ta}}$ respectively). Defect structures were obtained by relaxing the internal coordinates of a $3 \times 3 \times 1$ (near-cubic) expansion of the (relaxed) conventional tetragonal cell, consisting of 72 atoms when no defect is present, using a $6 \times 6 \times 5 \mathrm{k}$ mesh. The formation energy of defect $\mathrm{X}^{q}$ (in charge state $q$ ), $E_{f}\left[\mathrm{X}^{q}\right]$, assuming thermodynamic equilibrium, was determined from the equation

$$
E_{f}\left[\mathrm{X}^{q}\right]=E\left[\mathrm{X}^{q}\right]-E\left[\operatorname{bulk}^{q}\right]-\sum_{i} n_{i}\left(E_{i}+\Delta \mu_{i}\right)+q \Delta \phi,
$$

where $E\left[\right.$ bulk $\left.^{q}\right]$ is the total energy of the pure TaAs bulk supercell in charge state $q, E\left[\mathrm{X}^{q}\right]$ is the total energy of the supercell containing $\mathrm{X}^{q}, n_{i}$ is the number of species $i$ that is added to $\left(n_{i}>0\right)$ or removed from $\left(n_{i}<0\right)$ the supercell in forming $\mathrm{X}^{q}, E_{i}$ is the total energy of the elemental form of species $i, \Delta \mu_{i}$ is the chemical potential of species $i$, and $\Delta \phi$ is the change in Fermi level due to the introduction of $\mathrm{X}^{q}$. The $\Delta \mu_{i}$ are determined for As-rich and As-poor growth conditions by taking into account the formation enthalpies of the competing phases, as implemented in the CPLAP [35] code. To test convergence we have calculated $E_{f}\left[\mathrm{~V}_{\mathrm{Ta}}^{0}\right]$ using a $4 \times 4 \times 2$ expansion of the conventional cell, finding that the formation energy differs from that using the $3 \times 3 \times 1$ supercell by under $3 \%$, indicating that our chosen supercell is satisfactorily converged.

For charged defects, Eq. (1) does not include Makov-Paynetype correction terms [36], and therefore differs from that usually adopted for semiconductors [37-39]. Such corrections are introduced to account for periodic image charge interactions in systems with insulating properties and dielectric screening, which are calculated using lattice sums in the presence of a neutralizing charge background. By postulating a band gap for the homogeneous electron-hole gas (jellium), both the defect formation energy in a given charge state, with respect to the ideal system in this charge state, and the energy of ionization of the defect to another charge state can be calculated. For metals (and semimetals), similar ideas could be pursued, but considering that the electron-hole gas would have a zero energy gap, the fate of the ionized charge carriers must be treated explicitly. Whereas their charge distributions will be quite complex, their energy will be very close to the Fermi level in the infinite dilution and athermal limits. Consequently, we have determined the single point energies of bulk supercells containing the appropriate charge $\left(E\left[\mathrm{bulk}^{q}\right]\right)$ and used these as the reference energies for the charged defect supercells.
On the other hand, the interaction between the defect of interest and its periodic images will be efficiently screened by the zero-gap delocalized charge distribution. Indeed, we have tested the possibility of charge localization on the formation of point defects, finding in all cases that the delocalized case is significantly lower in energy. Moreover, for the case of $\mathrm{V}_{\mathrm{Ta}}^{0}$, which, on formation, leaves three holes in the system, we have tested possible localization by employing a hybrid density functional (HSE06 [40]), which counters the wellknown self-interaction error in DFT. We find that even with this functional, the solution with charge completely delocalized is lowest in energy (and the computed formation energy differs from that determined using PBEsol by less than $0.05 \mathrm{eV}$ ). The resulting procedure is strongly reminiscent of the approach adopted for charged defects in semiconductors and insulators when calculations on sufficiently large supercells were not feasible $[41,42]$.

In Eq. (1), $\Delta \phi$ is a parameter, giving the relation between formation energy and Fermi level. For a set of charged defects, however, one can compute $\Delta \phi$ in a self-consistent manner given the condition of charge neutrality in the system [43]:

$$
n_{0}+\sum_{i}\left|q_{i}\right| N_{\mathrm{A}_{i} q_{i}}=p_{0}+\sum_{i}\left|q_{i}\right| N_{\mathrm{D}_{i} q_{i}}
$$

where $n_{0}$ is the concentration of electrons, $N_{\mathrm{A}_{i} q_{i}}\left(N_{\mathrm{D}_{i} q_{i}}\right)$ the concentration of acceptors $\mathrm{A}_{i}$ (donors $\mathrm{D}_{i}$ ) in charge state $q_{i}$, and $p_{0}$ is the concentration of holes. All these concentrations are functions of $\Delta \phi$ as follows:

$$
\begin{aligned}
N_{\mathrm{X}^{q}} & =N_{0} \exp \left[E_{f}\left[\mathrm{X}^{q}\right](\Delta \phi) / k T\right], \\
n_{0} & =\int_{0}^{\infty} \frac{\exp (\Delta \phi / k T)}{1+\exp \left(E^{\prime} / k T\right)} d E^{\prime} \rho\left(E^{\prime}\right), \\
p_{0} & =\int_{-\infty}^{0} \frac{\exp (-\Delta \phi / k T)}{1+\exp \left(-E^{\prime} / k T\right)} d E^{\prime} \rho\left(E^{\prime}\right),
\end{aligned}
$$

where $N_{0}$ is the density of sites where $\mathrm{X}^{q}$ can be formed and $k$ is Boltzmann's constant. Therefore a self-consistent $\Delta \phi$ can be determined, as well as the electron, hole, and defect concentrations, given the set of $E_{f}$ and $\rho(E)$. Moreover, by fixing particular values of $N_{X^{q}}$, it is possible to determine $\Delta \phi$ for given defect concentrations. We have implemented this approach in the FORTRAN code SC-FERMI [44-46]. The Fermi level in the system $E_{F}$ is given by $E_{F}=E_{F_{0}}+\Delta \phi$.

Results. To determine how growth conditions will affect defect formation, we have calculated the phase stability of the stoichiometric phases $\mathrm{TaAs}_{2}, \mathrm{TaAs}, \mathrm{Ta}_{5} \mathrm{As}_{4}, \mathrm{Ta}_{2} \mathrm{As}$, and $\mathrm{Ta}_{3} \mathrm{As}$ as a function of $\Delta \mu_{\mathrm{As}}$ and $\Delta \mu_{\mathrm{Ta}}$; our results are shown in Fig. 1. The thick, solid lines indicate where the chemical potentials of Ta, As, and the relevant phase are in equilibrium, while the shaded regions indicate where each phase is the most stable and where an excess of Ta and/or As exists. In the inset of Fig. 1 we present the heats of formation for each phase with respect to elemental Ta and As (in parentheses within the boxes), and the heats of formation when transforming one phase to another by reacting with the relevant proportions of Ta and As (along the arrows). In all cases these heats of formation are negative, indicating exothermic reactions, but we find that $\mathrm{Ta}_{2} \mathrm{As}$ is thermodynamically unstable, i.e., it is a metastable phase that decomposes to $\mathrm{Ta}_{5} \mathrm{As}_{4}$ and $\mathrm{Ta}_{3} \mathrm{As}$ in proportions that depend 


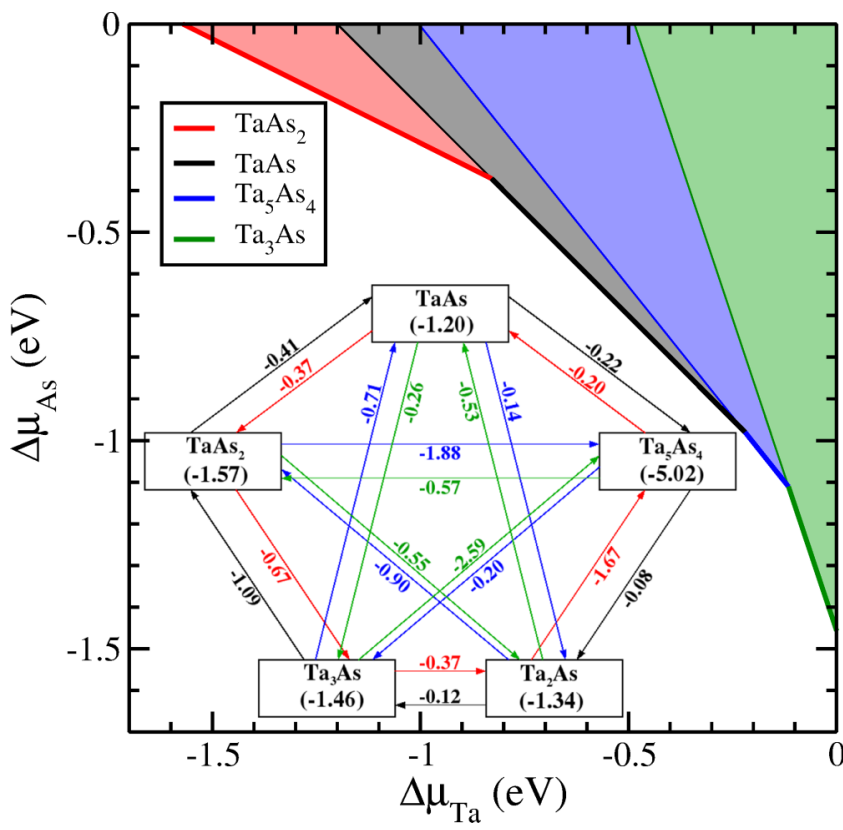

FIG. 1. Regions of stability within the phase space spanned by the Ta and As chemical potentials ( $\Delta \mu_{\mathrm{Ta}}$ and $\Delta \mu_{\mathrm{As}}$, respectively). The solid line represents the equilibrium chemical potentials at which each phase are most stable, while the shaded regions indicate the regions in which each phase is most favorable, while also favoring an excess of Ta and/or As. The inset shows the calculated heats of formation (in $\mathrm{eV}$ ), with the heat of formation of each stoichiometry, with respect to elemental Ta and As, shown in parentheses.

on environmental conditions, a result that is consistent with experimental findings [20]. The region in which TaAs is most favorable to form is mostly As rich (i.e., less negative $\Delta \mu_{\mathrm{As}}$ ), in agreement with experimental growth conditions [24].

To study point defect formation in TaAs, we have chosen two extreme conditions: As rich and As poor (where the thick black line meets the red and blue lines, respectively, in Fig. 1). Our calculated formation energies are presented in Fig. 2.
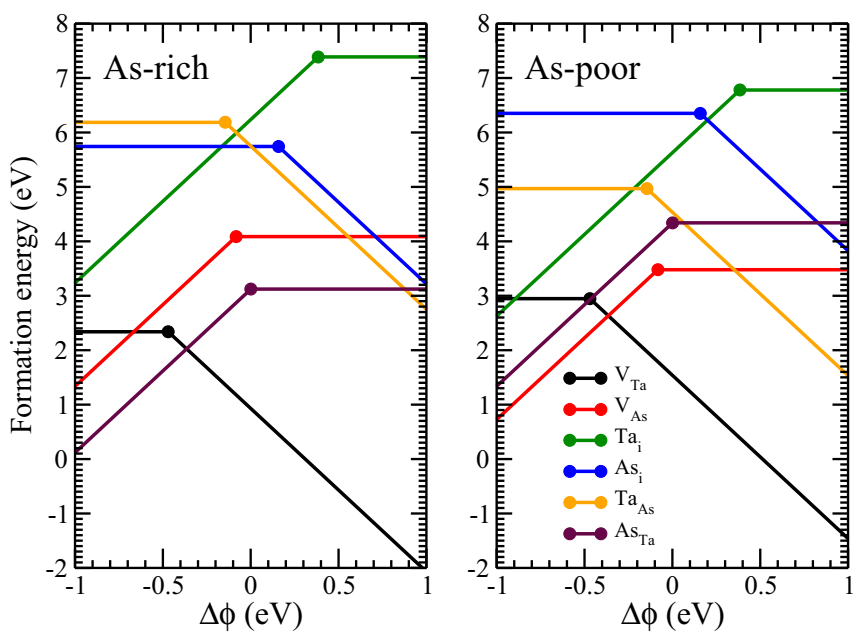

FIG. 2. Formation energies as a function of change in Fermi energy $(\Delta \phi)$ for each intrinsic defect studied, shown for As-rich and As-poor conditions. The slopes indicate the charge states; a transition occurs where the slope of a line changes.
In both cases, at the equilibrium Fermi level $(\Delta \phi=0)$, we find that $\mathrm{V}_{\mathrm{Ta}}^{3-}$ dominate. In As-rich conditions, the next-lowest energy defects are $\mathrm{As}_{\mathrm{Ta}}^{3+}$, which become competitive with Ta vacancies at $\Delta \phi \approx-0.35 \mathrm{eV}$, indicating a drive to form $\mathrm{TaAs}_{2}$, consistent with the phase diagram shown in Fig. 1. In As-poor conditions, Ta vacancies become less stable while As vacancies are more favorable, as one would expect. Our calculated formation energies of $\mathrm{V}_{\mathrm{Ta}}^{0}$ in As-poor conditions (2.95 eV) and $\mathrm{V}_{\mathrm{As}}^{0}$ in As-rich conditions (4.09 eV) are in excellent agreement with the work of Besara et al. [23] who used a smaller supercell $(2 \times 2 \times 1$ expansion of the conventional cell), further demonstrating that our calculations are well converged with respect to supercell size.

The formation energies shown in Fig. 2, however, are quite high for $\Delta \phi \approx 0$ (above $\sim 0.9 \mathrm{eV}$ ), meaning that equilibrium defect concentrations will be extremely low. Indeed, using the calculated formation energies to determine the self-consistent Fermi level for equilibrium As-rich conditions, and consequently the defect concentrations, we find that $N_{\mathrm{V}_{\mathrm{Ta}}}=1.6 \times$ $10^{7} \mathrm{~cm}^{-3}$ at temperature $T=300 \mathrm{~K}$. Such a concentration corresponds practically to perfect stoichiometry $\left(\delta<10^{-10}\right.$ in $\mathrm{Ta}_{\delta} \mathrm{As}$ ), which would disagree with the reported values of $\sim 0.92 \leqslant \delta \leqslant 0.98[21,23]$. If we include defects that form at typical synthesis temperatures $\left(T=1000^{\circ} \mathrm{C}\right.$ [24]) in an

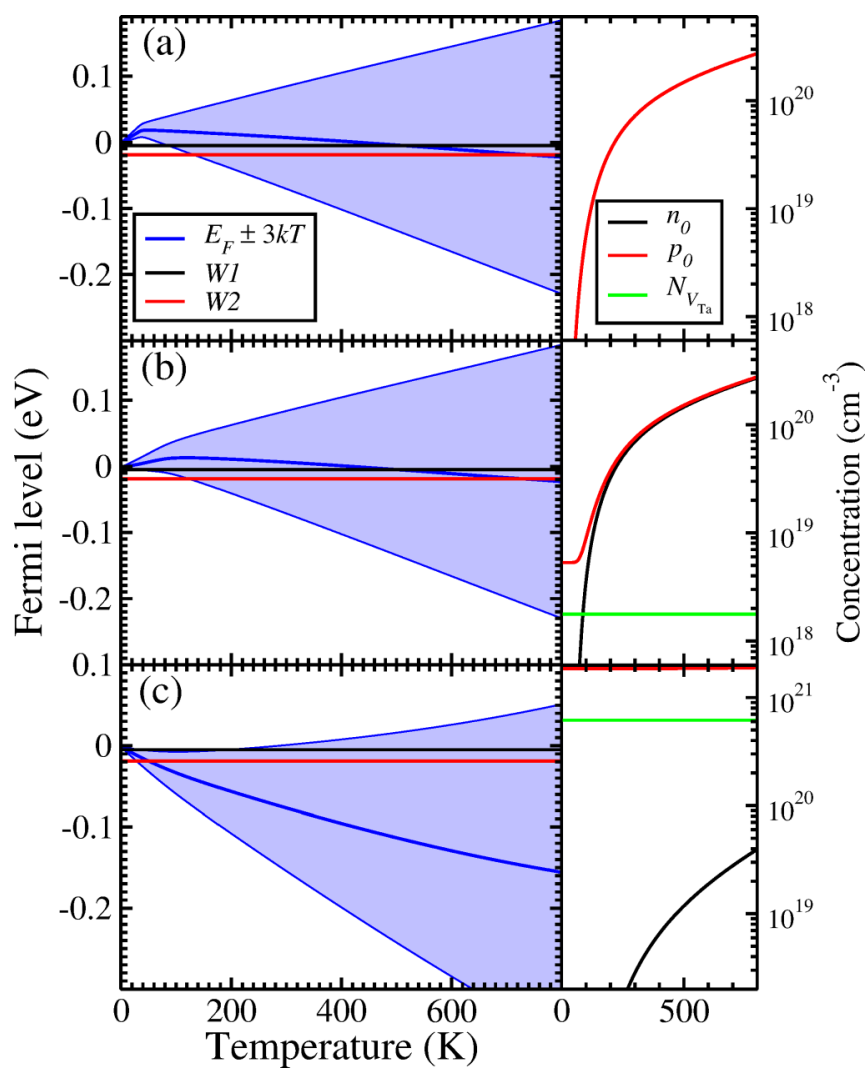

FIG. 3. Calculated variation in Fermi level $E_{F}$ (blue line, $k$ is the Boltzmann constant) as a function of temperature $T$ for (a) perfect stoichiometry, (b) slightly off perfect stoichiometry $(\delta=0.99994$ in $\mathrm{Ta}_{\delta} \mathrm{As}$ ), and (c) $\delta=0.98$. For each case the panel on the right shows the variation in electron $\left(n_{0}\right)$, hole $\left(p_{0}\right)$, and Ta vacancy $\left(N_{\mathrm{V}_{\mathrm{Ta}}}\right)$ concentrations with temperature. The positions of the $W 1$ and $W 2$ Weyl points are shown as black and red lines, respectively. 

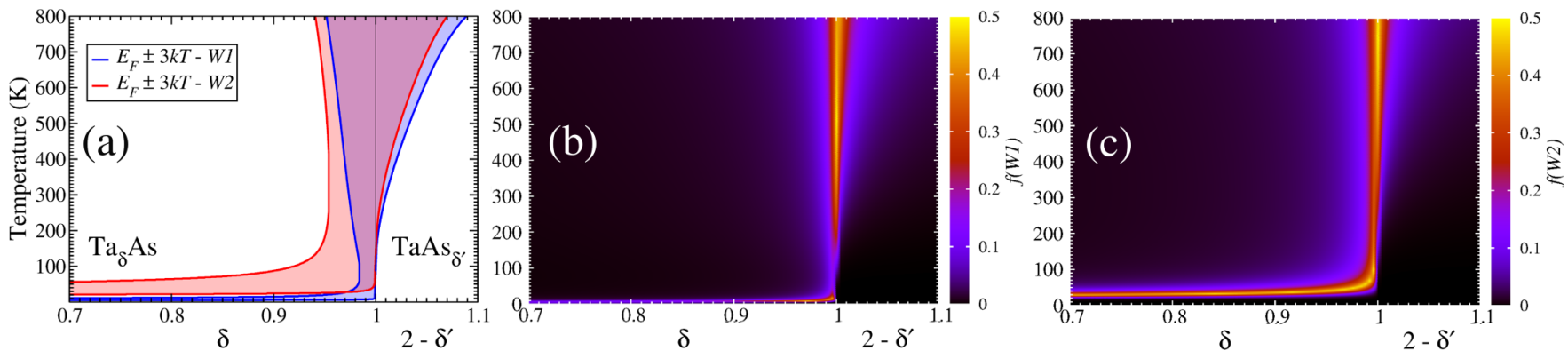

FIG. 4. Overlap of charge carrier energies and the Weyl points $W 1$ and $W 2$ as a function of nonstoichiometry $\left(\delta\right.$ in $\mathrm{Ta}_{\delta} \mathrm{As}$ and $\delta^{\prime}$ in $\left.\mathrm{TaAs}_{\delta^{\prime}}\right)$ and temperature $T$. The overlap is shown as (a) the regions in which $E_{F} \pm 3 k T-W$ is positive ( $k$ is Boltzmann's constant), where $W=W 1$ (blue) or $W 2$ (red); and the value of the Fermi function at (b) $W 1(f(W 1))$ and (c) $W 2(f(W 2))$.

equilibrium process and get "frozen in" by kinetic barriers on cooling, we find that $N_{\mathrm{V}_{\mathrm{Ta}}}=1.8 \times 10^{18} \mathrm{~cm}^{-3}$, corresponding to $\delta=0.99994$, again disagreeing with observed values of $\delta$. Our results demonstrate that intrinsic defect formation at thermodynamic equilibrium is not the source of nonstoichiometry; instead the particular synthetic route, including annealing processes, accounts for the defect concentrations, which, according to our calculations will be dominated by vacancies. The fact that the decomposition of $\mathrm{TaAs}_{2}$ is a practical source of TaAs preparation [19], a favorable process according to Fig. 1, also suggests large vacancy concentrations can be frozen in. We can thus simulate observed levels of nonstoichiometry by setting $N_{\mathrm{V}_{\mathrm{Ta}}}=6.2 \times 10^{20} \mathrm{~cm}^{-3}$, which gives a value of $\delta=0.98$, consistent with various experimental studies.

In Fig. 3 our calculated self-consistent Fermi level as a function of $T$ is shown for (a) $\delta=1$, (b) $\delta=0.99994$, and (c) $\delta=0.98$, with the associated variation in $n_{0}, p_{0}$, and $N_{\mathrm{V}_{\mathrm{Ta}}}$ with $T$ shown in the right-hand panels [for Figs. 3(b) and 3(c), $N_{\mathrm{V}_{\mathrm{Ta}}}$ is held constant to simulate frozen-in concentrations]. We also show the positions of the Weyl nodes $W 1$ and $W 2$ relative to $E_{F}$ (which were determined to be at $E_{F_{0}}-0.005 \mathrm{eV}$ and $E_{F_{0}}-0.019 \mathrm{eV}$ respectively [34]), and show the range $E_{F} \pm 3 k T$ ( $k$ is Boltzmann's constant), which corresponds to the range in which probability of overlap will be greater than $\sim 0.05$. Our results demonstrate that for pure TaAs, Weyl effects will not be observed clearly for $T \lesssim 120 \mathrm{~K}$, because both $W 1$ and $W 2$ do not lie within $3 k T$ of $E_{F}$. Moving slightly off stoichiometry reduces this cutoff temperature to $T \lesssim 90 \mathrm{~K}$ [see Fig. 3(b)], but going to more realistic values of $\delta=0.98$ opens up the range in which the effects can be observed greatly. This delicate balance in observability as a function of nonstoichiometry is quite surprising and indicates a degree of fortuity in the discovery of Weyl fermions in TaAs.

We finally show where observability of Weyl effects is likely within the space spanned by nonstoichiometry $(\delta$ in $\mathrm{Ta}_{\delta}$ As and $\delta^{\prime}$ in $\mathrm{TaAs}_{\delta^{\prime}}$ ) and $T$ by showing in Fig. 4(a) the regions in which $W 1$ and $W 2$ are within $3 k T$ of $E_{F}$, and in Figs. 4(b) and 4(c) the value of the Fermi function at $W 1$ and $W 2$ respectively, where $f(W)=\{1+\exp [ \pm(W-$ $\left.\left.\left.E_{F}\right) / k T\right]\right\}^{-1}(W=W 1$ or $W 2$ and the sign of the exponent depends on whether the charge carriers are electrons or holes). We see that there is a narrow band of nonstoichiometry in which observation is possible for a wide range of temperatures. In As-poor samples, observability at $T \lesssim 300 \mathrm{~K}$ becomes unlikely quite rapidly, while in Ta-poor samples, for $\delta<0.95$ observability is only possible within a restricted temperature range below $100 \mathrm{~K}$.

Discussion. Of course, our approach assumes the condition of thermodynamical equilibrium for the observation of Weyl effects. Experimental techniques that allow access to unoccupied states [47], e.g., hot carrier injection methods or excitation via ionizing radiation, may allow such observations in certain conditions, in regions deemed unlikely by our calculations. Importantly, however, ARPES, the key technique used to observe Weyl fermion effects [4], does not permit this access to unoccupied states (although deep occupied states can be probed, such states do not contribute to the surface Fermi arcs that are characteristic of Weyl fermions). Moreover, we have not considered here the robustness of the Weyl nodes in the presence of defects. In fact, defects may adversely affect the presence of these band structure features [23], meaning the range of observability shown in Fig. 4 could be significantly reduced.

Our results show that small changes in stoichiometry can have large effects on the temperature ranges within which exotic condensed-matter physics phenomena can be observed. Indeed, the experimental conditions of $T=300 \mathrm{~K}$ and $\delta=$ $0.98[21,23,24]$ fall within the narrow region in which both $W 1$ and $W 2$ are likely to be observed, while moving away from these conditions has a drastic effect on observability. We postulate that such a situation may be widespread in samples under investigation for novel physical properties, whether these be double Weyl semimetals [48], topological insulators [49], or high- $T$ superconductors [50]. Our approach adopted here should therefore provide an avenue of exploration for future studies of such systems.

Summary. We have calculated the energy overlap of charge carriers with the Weyl nodes in TaAs as a function of temperature and nonstoichiometry, using first-principles calculations of the intrinsic point defect formation energies and their variation with Fermi level. Our results show that nonstoichiometry is not only compatible with, but necessary in a range of experimental conditions for, the observation of Weyl effects, indicating a serendipitous discovery. This approach can be applied to other systems displaying exotic physical phenomena that depend crucially on the Fermi level and will offer insights into the experimental conditions necessary for their observation.

Acknowledgments. J.B. and C.R.A.C. acknowledge funding from EPSRC Grant No. EP/K016288/1. The authors acknowledge the use of the UCL Legion and Grace 
High Performance Computing Facilities (Legion@UCL and Grace@UCL) and associated support services and the ARCHER supercomputer through membership of the UK's
HPC Materials Chemistry Consortium, which is funded by EPSRC Grant No. EP/L000202, in the completion of this work.
[1] P. A. M. Dirac, Proc. R. Soc. London A 117, 610 (1928).

[2] H. Weyl, Z. Phys. 56, 330 (1929).

[3] P. B. Pal, Am. J. Phys. 79, 485 (2011).

[4] S.-Y. Xu, I. Belopolski, N. Alidoust, M. Neupane, G. Bian, C. Zhang, R. Sankar, G. Chang, Z. Yuan, C.-C. Lee, S.-M. Huang, H. Zheng, J. Ma, D. S. Sanchez, B. Wang, A. Bansil, F. Chou, P. P. Shibayev, H. Lin, S. Jia, and M. Z. Hasan, Science 349, 613 (2015).

[5] H. Weng, C. Fang, Z. Fang, B. A. Bernevig, and X. Dai, Phys. Rev. X 5, 011029 (2015).

[6] I. Belopolski, S.-Y. Xu, D. S. Sanchez, G. Chang, C. Guo, M. Neupane, H. Zheng, C.-C. Lee, S.-M. Huang, G. Bian, N. Alidoust, T.-R. Chang, B. K. Wang, X. Zhang, A. Bansil, H.-T. Jeng, H. Lin, S. Jia, and M. Z. Hasan, Phys. Rev. Lett. 116, 066802 (2016).

[7] S.-M. Huang, S.-Y. Xu, I. Belopolski, C.-C. Lee, G. Chang, B. Wang, N. Alidoust, G. Bian, M. Neupane, C. Zhang, S. Jia, A. Bansil, H. Lin, and M. Z. Hasan, Nat. Commun. 6, 7373 (2015).

[8] L. X. Yang, Z. K. Liu, Y. Sun, H. Peng, H. F. Yang, T. Zhang, B. Zhou, Y. Zhang, Y. F. Guo, M. Rahn, D. Prabhakaran, Z. Hussain, S.-K. Mo, C. Felser, B. Yan, and Y. L. Chen, Nat. Phys. 11, 728 (2015).

[9] B. Q. Lv, H. M. Weng, B. B. Fu, X. P. Wang, H. Miao, J. Ma, P. Richard, X. C. Huang, L. X. Zhao, G. F. Chen, Z. Fang, X. Dai, T. Qian, and H. Ding, Phys. Rev. X 5, 031013 (2015).

[10] B. Q. Lv, N. Xu, H. M. Weng, J. Z. Ma, P. Richard, X. C. Huang, L. X. Zhao, G. F. Chen, C. E. Matt, F. Bisti, V. N. Strocov, J. Mesot, Z. Fang, X. Dai1, T. Qian, M. Shi, and H. Ding, Nat. Phys. 11, 724 (2015).

[11] B. Q. Lv, S. Muff, T. Qian, Z. D. Song, S. M. Nie, N. Xu, P. Richard, C. E. Matt, N. C. Plumb, L. X. Zhao, G. F. Chen, Z. Fang, X. Dai, J. H. Dil, J. Mesot, M. Shi, H. M. Weng, and H. Ding, Phys. Rev. Lett. 115, 217601 (2015).

[12] Z. K. Liu, L. X. Yang, Y. Sun, T. Zhang, H. Peng, H. F. Yang, C. Chen, Y. Zhang, Y. F. Guo, D. Prabhakaran, M. Schmidt, Z. Hussain, S.-K. Mo, C. Felser, B. Yan, and Y. L. Chen, Nat. Mater. 15, 27 (2016).

[13] N. Xu et al., Nat. Commun. 7, 11006 (2016).

[14] S.-Y. Xu et al., Nat. Phys. 11, 748 (2015).

[15] H. Zheng et al., ACS Nano 10, 1378 (2016).

[16] X. Huang, L. Zhao, Y. Long, P. Wang, D. Chen, Z. Yang, H. Liang, M. Xue, H. Weng, Z. Fang, X. Dai, and G. Chen, Phys. Rev. X 5, 031023 (2015).

[17] F. Arnold et al., Nat. Commun. 7, 11615 (2016).

[18] H. Boller and E. Parthé, Acta Cryst. 16, 1095 (1963).

[19] S. Furuseth, K. Selte, and A. Kjekshus, Acta Chem. Scand. 19, 95 (1965).

[20] J. J. Murray, J. B. Taylor, L. D. Calvert, Y. Wang, E. J. Gabe, and J. G. Despault, J. Less Common Met. 46, 311 (1976).

[21] B. Saparov, J. E. Mitchell, and A. S. Sefat, Supercond. Sci. Technol. 25, 084016 (2012).
[22] Y. Liu, Z. Li, L. Guo, X. Chen, Y. Yuan, F. Liu, S. Prucnal, M. Helm, and S. Zhou, J. Magn. Magn. Mater. 408, 73 (2016).

[23] T. Besara, D. A. Rhodes, K.-W. Chen, S. Das, Q. R. Zhang, J. Sun, B. Zeng, Y. Xin, L. Balicas, R. E. Baumbach, E. Manousakis, D. J. Singh, and T. Siegrist, Phys. Rev. B 93, 245152 (2016).

[24] Z. Li, H. Chen, S. Jin, D. Gan, W. Wang, L. Guo, and X. Chen, Cryst. Growth Des. 16, 1172 (2016)

[25] G. Kresse and J. Hafner, Phys. Rev. B 47, 558 (1993).

[26] G. Kresse and J. Hafner, Phys. Rev. B 49, 14251 (1994).

[27] G. Kresse and J. Furthmüller, Comput. Mater. Sci. 6, 15 (1996).

[28] G. Kresse and J. Furthmüller, Phys. Rev. B 54, 11169 (1996).

[29] J. P. Perdew, K. Burke, and M. Ernzerhof, Phys. Rev. Lett. 77, 3865 (1996).

[30] J. P. Perdew, A. Ruzsinszky, G. I. Csonka, O. A. Vydrov, G. E. Scuseria, L. A. Constantin, X. Zhou, and K. Burke, Phys. Rev. Lett. 100, 136406 (2008).

[31] P. E. Blöchl, Phys. Rev. B 50, 17953 (1994).

[32] H. J. Monkhorst and J. D. Pack, Phys. Rev. B 13, 5188 (1976).

[33] D. Hobbs, G. Kresse, and J. Hafner, Phys. Rev. B 62, 11556 (2000).

[34] J. Buckeridge, D. Jevdokimovs, C. R. A. Catlow, and A. A Sokol, Phys. Rev. B 93, 125205 (2016).

[35] J. Buckeridge, D. O. Scanlon, A. Walsh, and C. R. A. Catlow, Comput. Phys. Commun. 185, 330 (2014).

[36] G. Makov and M. C. Payne, Phys. Rev. B 51, 4014 (1995).

[37] S. Lany and A. Zunger, Phys. Rev. B 78, 235104 (2008).

[38] C. Freysoldt, J. Neugebauer, and C. G. Van de Walle, Phys. Status Solidi B 248, 1067 (2011).

[39] H.-P. Komsa, T. T. Rantala, and A. Pasquarello, Phys. Rev. B 86, 045112 (2012).

[40] J. Heyd, G. E. Scuseria, and M. Ernzerhof, J. Chem. Phys. 124, 219906 (2006).

[41] A. S. Foster, F. Lopez Gejo, A. L. Shluger, and R. M. Nieminen, Phys. Rev. B 65, 174117 (2002).

[42] J. L. Gavartin, A. L. Shluger, A. S. Foster, and G. I. Bersuker, J. Appl. Phys. 97, 053704 (2005).

[43] C. Kittel and H. Kroemer, Thermal Physics, 2nd ed. (Freeman, San Francisco, 1980), Chap. 13.

[44] https://sourceforge.net/projects/sc-fermi.

[45] https://github.com/projects/sc-fermi.git.

[46] F. H. Taylor, J. Buckeridge, and C. R. A. Catlow, Chem. Mater. (2016), doi:10.1021/acs.chemmater.6b03048.

[47] X.-X. Zhang, T. T. Ong, and N. Nagaosa, arXiv:1607.05941.

[48] S.-M. Huang et al., Proc. Natl. Acad. Sci. U.S.A. 113, 1180 (2016).

[49] A. Bansil, H. Lin, and T. Das, Rev. Mod. Phys. 88, 021004 (2016).

[50] B. Keimer, S. A. Kivelson, M. R. Norman, S. Uchida, and J. Zaanen, Nature 518, 179 (2015). 